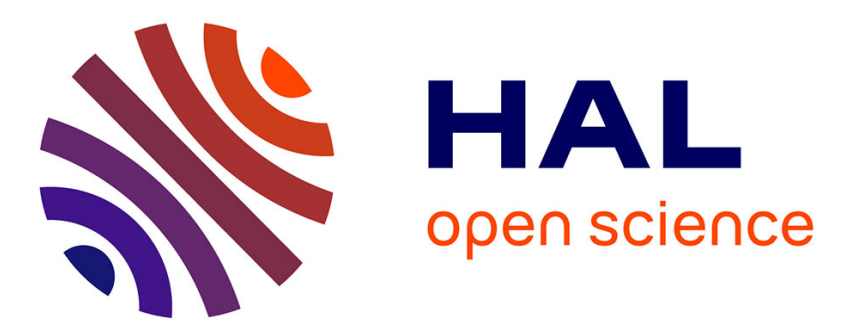

\title{
A new hybrid next-event estimator for photon-based Monte Carlo dose rate calculations
}

Ettore Guadagni, Cindy Le Loirec, Yannick Pénéliau, Jean Michel Létang, Davide Mancusi, Cheikh Diop

\section{- To cite this version:}

Ettore Guadagni, Cindy Le Loirec, Yannick Pénéliau, Jean Michel Létang, Davide Mancusi, et al.. A new hybrid next-event estimator for photon-based Monte Carlo dose rate calculations. The European Physical Journal Plus, 2021, 136 (11), pp.1135. 10.1140/epjp/s13360-021-02120-5 . hal-03442633

\section{HAL Id: hal-03442633 \\ https://hal.science/hal-03442633}

Submitted on 3 Dec 2021

HAL is a multi-disciplinary open access archive for the deposit and dissemination of scientific research documents, whether they are published or not. The documents may come from teaching and research institutions in France or abroad, or from public or private research centers.
L'archive ouverte pluridisciplinaire HAL, est destinée au dépôt et à la diffusion de documents scientifiques de niveau recherche, publiés ou non, émanant des établissements d'enseignement et de recherche français ou étrangers, des laboratoires publics ou privés. 


\title{
A new hybrid next-event estimator for photon-based Monte Carlo dose rate calculations
}

\author{
Ettore Guadagni ${ }^{1}$, Cindy Le Loirec ${ }^{1}$, Yannick Pénéliau ${ }^{2}$, Jean Michel Létang ${ }^{3}$, Davide Mancusi ${ }^{4}$ and $_{\text {Cheikh Diop }}{ }^{4}$ \\ 1 CEA, DES, IRESNE, DER, Service de Physique des Réacteurs et du Cycle, Cadarache F-13108 Saint-Paul-lez-Durance, \\ France \\ 2 CEA, IRFM, Cadarache F-13108 Saint-Paul-Lez-Durance, France \\ 3 Univ Lyon, INSA-Lyon, Université Claude Bernard Lyon 1, Centre Léon Bérard, CNRS, Inserm, CREATIS UMR 5220 U1206, \\ 69373, Lyon, France \\ ${ }^{4}$ Université Paris-Saclay, CEA, Service d'Etudes des Réacteurs et de Mathématiques Appliquées, 91191, Gif-sur-Yvette, France
}

Received: date / Revised version: date

\begin{abstract}
This work describes the implementation of a deterministic-like straight-line transport routine inside the TRIPOLI- $4^{\circledR}$ Monte Carlo code, aimed at developing a hybrid estimator that can speed-up the $\mathrm{MC}$ calculation while retaining its precision. The algorithm is based on the exponential track length estimator or $e \mathrm{TLE}$, a next-event estimator that has already been investigated for medical applications. In this implementation, the $e$ TLE is paired with a forced detection algorithm which is shown to drastically improve its performance. We describe the implementation of the method in TRIPOLI- ${ }^{\circledR}$, then we present the first results obtained over some test cases and compare them with the classic track-length estimator already implemented in TRIPOLI- $4{ }^{\circledR}$.
\end{abstract}

\section{Introduction}

When planning the decommissioning and dismantling of a nuclear facility, dose rate estimation constitutes a crucial step to define operational margins and to ensure that safety limits are respected. To this aim, one can distinguish two main families of computer codes:

- The deterministic ones, like RANKERN [1] and CEA's NARMER [2], are often based on Point-Kernel Integration (PKI) method, with build-up and/or albedo factors. PKI is a macroscopic approach that consists in considering any radiation source as an ensemble of independent beam-like point sources. These straight beams of radiation are attenuated through matter by the means of macroscopic coefficients, such as the linear attenuation coefficient. The build-up and albedo factors are correction factors used to estimate the effect of scattering in matter and surface reflection, respectively.

- The stochastic ones, like MCNP [3], GEANT4 [4] and CEA's TRIPOLI-4 ${ }^{\circledR}$ [5], are based on Monte Carlo (MC) method. This technique is a microscopic approach that can numerically evaluate arbitrarily complex stochastic processes by starting from basic known probability distributions. In the particle transport field, these distributions are based on isotopic data and the stochastic process is the random-walk of single particles, mainly photons in the case of dismantling applications.

Deterministic PKI-based codes are inherently fast from a computational point of view, as they calculate the dose purely by analytical means, by exponentially attenuating gammas on a straight-line path and integrating over the radiation source volume. This allows the PKI method to be used in real-time dose computation tools like Visiplan [6] and Mercurad [7], and opens the door to virtual reality implementation [8]. However, the speed of the PKI method comes at the cost of accuracy; the analytical dose calculations are based on several assumptions and simplifications which may break down in specific situations, such as scattering-dominated problems, or in the case of very short distance between radiation source and detector [9].

In contrast, MC codes enable a precise estimation of gamma dose rate and can converge to any user-specified level of confidence. However, the determination of local equivalent dose rate needs a very large number of gamma histories to achieve reliable results, which lead to long computation times. For large geometries (i.e. reactor-level calculations) the computational burden becomes unfeasible for practical use. Luckily, there exists a large number of options to speed up any MC calculation, in the form of variance reduction techniques (VRTs) [10]. These techniques consist in modifying 
the analog random sampling procedure to focus computational efforts on the simulation of important particles, i.e. particles that are expected to contribute more to the solution [11].

Alongside VRTs, another way of enhancing the efficiency of a MC simulation is the correct choice of the estimator. It has actually been shown [12] that the appropriate choice of an estimator can dramatically reduce the computation time needed to obtain a sufficiently small confidence interval about the MC estimate. The most basic estimator in MC simulations is the so-called "collision estimator", which simply estimates the particle flux in a volume by tallying all the interactions taking place inside the volume and weighting them by the inverse of the total macroscopic cross section. This estimator is simple and effective enough to be implemented in virtually every MC code. However, since it is intrinsically related to the number of collisions taking place in the estimation volume, its efficiency drops when it is used in low-density regions or in optically thin regions. In these instances, the collision estimator can produce unreliable estimates with very high variance. Another class of estimators, called track-length estimators (TLEs) can efficiently tackle such problems. TLEs are based on the equivalence between particle fluence and total particle path length per unit volume [13]. The gain in efficiency comes from the fact that, for this class of estimators, every particle trajectory intersecting the estimation volume contributes to the tally; it is easy to see how, for the same number of histories generated, the TLE will always have a number of contributions larger than or equal to the collision estimator. That is why this estimator, in its most simple implementation (called linear TLE or just TLE for short), is nowadays largely used in MC codes [14], and has shown good performance for low-energy applications in medical fields [15] and for reaction rate estimations in the nuclear field [16]. Starting from the classic formulation of the linear TLE, it is possible to derive a more sophisticated estimator called exponential track-length estimator or $e$ TLE. The main difference between TLE and $e$ TLE is that the latter replaces a stochastic decision, i.e. the free flight of the particle, by its expected outcome averaged over the probability distribution of particle flight lengths. Such replacement of a random decision by its expected outcome is a frequently used VRT [17]. In the eTLE, after each collision the particle is analytically transported in a straight line up until the simulation boundary, while being exponentially attenuated. It can therefore be viewed as a hybrid transport method, combining stochastic MC and deterministic straight-line transport. Starting from the idea that MC calculations of dose deposition could be accelerated by deterministic transport of photons, a new hybrid approach was developed by Freud et al. [18,19]. In this approach, a forced detection [20] algorithm was added to the method, transporting the photons in predefined directions to maximize efficiency. The proposed method, even if just in a very preliminary way, showed good results when compared to the classical MC approach with TLE. In a previous work [21], we described the first implementation of $e$ TLE in TRIPOLI-4 ${ }^{\circledR}$ for dismantling purposes. This first study highlighted the acceleration capabilities of the new estimator, which provided consistent results by reducing computation time by more than a half in the best cases, when compared to the classic TLE. In the same study the $e$ TLE was also coupled with two of TRIPOLI-4 ${ }^{\circledR}$ 's variance reduction techniques, the exponential transform and the advanced multilevel splitting, showing encouraging results. In this paper, we couple the $e$ TLE with a forced detection (FD) algorithm that automatically sends particles towards detectors, drastically improving the efficiency of the estimator in dealing with localized dose deposition calculations.

This process of sending a particle towards a detector to improve the simulation's efficiency shares some important similarities with some other algorithms employed in particle transport; notably, the next-event point estimator [22] on one side, and algorithms like DXTRAN [23] or the one developed by Tickner [24] on the other. However, the $e$ TLE with FD is fundamentally different from these approaches. The next-event point estimator, also present in TRIPOLI-4 $\mathbb{R}$ with the name FLUX PT, is a well known estimator in the field of MC simulations. As the name implies, it allows to estimate quantities in a point; it also involves deterministic transport and exponential attenuation of the particle's weight. In practice, it can be viewed as a limit version of the eTLE with FD, where the target detector becomes infinitely small, converging to a single point. As explained by Cramer in [22], this change from volume to point leads to the appearance, in the estimator, of a $1 / R^{2}$ term, where $R$ is the distance between the point of collision and the point of detection. This term causes the point estimator's variance to drastically diverge whenever a collision takes place very close to the point of detection, and it is the reason why every MC implementation of next-event point estimation usually includes an "exclusion sphere" inside of which any contribution to the estimator is not taken into account. Therefore, the main difference between the $e$ TLE with FD and a point estimator is that the former does not require any exclusion sphere. As for DXTRAN [23] and DXTRAN-like transport algorithms [24], the difference is more related to the mechanics of the particle. In these algorithms, the particle is split at every interaction, and deterministically transported to the surface of some element: a sphere in the case of DXTRAN, any convex volume in Tickner's implementation. Then, the transported particle is left free to continue its simulation, until it is absorbed or leaves the simulation domain. Therefore, the transported particle can, in principle, interact many times inside the volume of interest before ending its simulation history. This in turn means that the "mother" particle has to be killed if it tries to enter the volume of interest, as its interactions have already been simulated by the split particle. The fundamental difference between this approach and the $e$ TLE with FD is that, in the latter, there is no actual particle being transported towards the detection volume, but rather a "virtual" particle representing a single contribution of the mother particle to the score. This "virtual" particle gives one and only one contribution, and then it is killed, as will be better detailed in Section 2.2. Moreover, the "real" particle only serves as a mean to simulate collision points, 
but it never registers any score: all the contributions to the $e$ TLE with FD come from the "virtual" particles. This is also why, in our method, there is no need to kill the "real" particle if it tries to enter the detection volume.

In this paper, the new $e$ TLE with forced detection is tested on a series of simplified scenarios representative of decommissioning configurations. Its performances are compared with the simpler version of the $e$ TLE without forced detection and with the legacy TLE, as implemented in TRIPOLI- ${ }^{\circledR}$.

\section{Methodology}

\subsection{Theoretical basis of $e$ TLE}

The idea behind the family of TLEs is the following: all the particle flights that intersect the scoring volume (see fig. 1) contribute to the estimate. If we compare this approach to the collision estimator, that only tallies the interactions happening inside the scoring volume, any type of TLE can in principle lower the variance of the simulation, as this last set of events is always smaller than, or equal to, the former. Depending on the implementation, several types of track-length estimators exist; for the purpose of this discussion, we shall concentrate on the estimators for the particle flux.

In the linear formulation of TLE, the contribution of a flight is proportional to the length of the flight segment inside the scoring volume $V$. This fluence estimator would give a non-zero value after collisions 2,3 and 4 in fig. 1a, whereas a collision estimator would only register collision 4 . More specifically, the contribution to volume $V$ given to the linear TLE by a flight from $\boldsymbol{r}_{j}$ to $\boldsymbol{r}_{j+1}$ is equal to:

$$
K_{V}^{\mathrm{TLE}}\left(\boldsymbol{r}_{j}, \boldsymbol{r}_{j+1}\right)=w \frac{L_{V}\left(\boldsymbol{r}_{j}, \boldsymbol{r}_{j+1}\right)}{V},
$$

where $w$ is the weight of the particle and $L_{V}\left(\boldsymbol{r}_{j}, \boldsymbol{r}_{j+1}\right)$ is the straight-line distance traveled by the particle inside the scoring volume between the two successive interactions $\boldsymbol{r}_{j}$ and $\boldsymbol{r}_{j+1}$; more rigorously, we can write:

$$
L_{V}\left(\boldsymbol{r}_{j}, \boldsymbol{r}_{j+1}\right)=\int_{0}^{\left|\boldsymbol{r}_{j+1}-\boldsymbol{r}_{j}\right|} \Pi_{V}\left(\boldsymbol{r}_{j}+s \boldsymbol{\Omega}\right) d s
$$

where $\boldsymbol{\Omega}$ is the direction of the vector pointing from $\boldsymbol{r}_{j}$ to $\boldsymbol{r}_{j+1}$ and $\Pi_{V}(\boldsymbol{r})$ is the characteristic function of the scoring volume $V$ (which is equal to 1 if the point $r$ is inside the volume and equal to 0 otherwise).

We can derive the $e$ TLE from its linear correspondent by replacing the estimator in Eq. (1) with its average value over all possible flights lengths emerging from collision $j$ in the direction $\Omega$. As such, the $e$ TLE estimator is still a flight-based estimator (i.e. it contributes to the score every time a particle performs a flight), but its value does not depend on the end point of the flight $\boldsymbol{r}_{j+1}$ (only on the direction $\boldsymbol{\Omega}$ ). According to the definition, for the $e$ TLE we can write:

$$
K_{V}^{e \mathrm{TLE}}\left(\boldsymbol{r}_{j}, \boldsymbol{\Omega}\right)=\int_{0}^{\infty} K_{\mathrm{TLE}}\left(\boldsymbol{r}_{j}, \boldsymbol{r}_{j}+s \boldsymbol{\Omega}\right) \exp \left(-\int_{0}^{s} \Sigma_{t}\left(\boldsymbol{r}_{j}+t \boldsymbol{\Omega}\right) d t\right) d s,
$$

where $\Sigma_{t}(\boldsymbol{r})$ is the total macroscopic cross section at position $\boldsymbol{r}$. If the scoring volume $V$ is uniform $\left(\Sigma_{t}(\boldsymbol{r})\right.$ constant inside the detector) and convex, then the eTLE estimator can be rewritten in the simpler form

$$
K_{V}^{e \mathrm{TLE}}\left(\boldsymbol{r}_{j}, \boldsymbol{\Omega}\right)=\frac{w}{\Sigma_{t} V} \exp \left(-\int_{0}^{s_{V}} \Sigma_{t}\left(\boldsymbol{r}_{j}+s \boldsymbol{\Omega}\right) d s\right)\left[1-\exp \left(-\Sigma_{t} L_{V}\left(\boldsymbol{r}_{j}, \infty\right)\right)\right] ;
$$

where $s_{V}$ is the distance from $\boldsymbol{r}_{j}$ to the scoring volume $V$ along direction $\boldsymbol{\Omega}$, and $L_{V}\left(\boldsymbol{r}_{j}, \infty\right)$ is the length of the chord intersected by $V$ after collision in $\boldsymbol{r}_{j}$ along direction $\boldsymbol{\Omega}$, the expression of which can be obtained by Eq. (2) by extending to infinity the distance between $\boldsymbol{r}_{j}$ and $\boldsymbol{r}_{j+1}$ :

$$
L_{V}\left(\boldsymbol{r}_{j}, \infty\right)=\int_{0}^{\infty} \Pi_{V}\left(\boldsymbol{r}_{j}+s \boldsymbol{\Omega}\right) d s
$$

The efficiency of this method can be illustrated by fig. 1b, where we can see that the particle is not even required to cross the scoring volume to contribute to the score.

An additional improvement can be brought by observing that the $e$ TLE estimator can only contribute to the score if the line of flight intersects the scoring volume. By transforming the $e$ TLE estimator in a collision-based estimator, we can sample virtual collisions directed towards the detection volume and weight them by their probability of occurrence. Fig. 1c shows the additional improvement in efficiency obtained by adding a forced detection (FD) algorithm to the $e$ TLE. Assuming the detection volume is a sphere, we sample a uniformly random direction $\Omega$ under the constraint 
that the half line from the collision point $\boldsymbol{r}_{1}$ directed along $\Omega$ intersects the sphere. We can write the score for the $e$ TLE with FD as:

$$
K_{V}^{e \mathrm{TLE}, \mathrm{FD}}\left(\boldsymbol{r}_{1}, \boldsymbol{\Omega}\right)=\frac{w}{\Sigma_{t} V} \exp \left(-\int_{0}^{s_{V}} \Sigma_{t}\left(\boldsymbol{r}_{1}+s^{\prime} \boldsymbol{\Omega}\right) d s^{\prime}\right)\left(1-\exp \left(-\Sigma_{t} L\right)\right) p\left(\boldsymbol{r}_{1}, \boldsymbol{\Omega}\right) \Delta \Omega
$$

where $p\left(\boldsymbol{r}_{1}, \boldsymbol{\Omega}\right)$ is the probability density, per unit solid angle, of scattering in the direction $\Omega$ after an interaction at $\boldsymbol{r}_{1}$, and $\Delta \Omega$ is the solid angle of the scoring volume seen from the interaction point. If the detection volume is not spherical, the solid angle can be hard to calculate; however, the problem is easily solved by defining a bounding sphere surrounding the volume and then scoring only the particles that effectively cross the volume, as shown in fig. 1c. In this case, the solid angle of the bounding sphere can be easily calculated by knowing the sphere radius $R$ and the distance $D$ between the interaction point and the center of the sphere:

$$
\Delta \Omega=2 \pi\left(1-\frac{\sqrt{D^{2}-R^{2}}}{D^{2}}\right)
$$

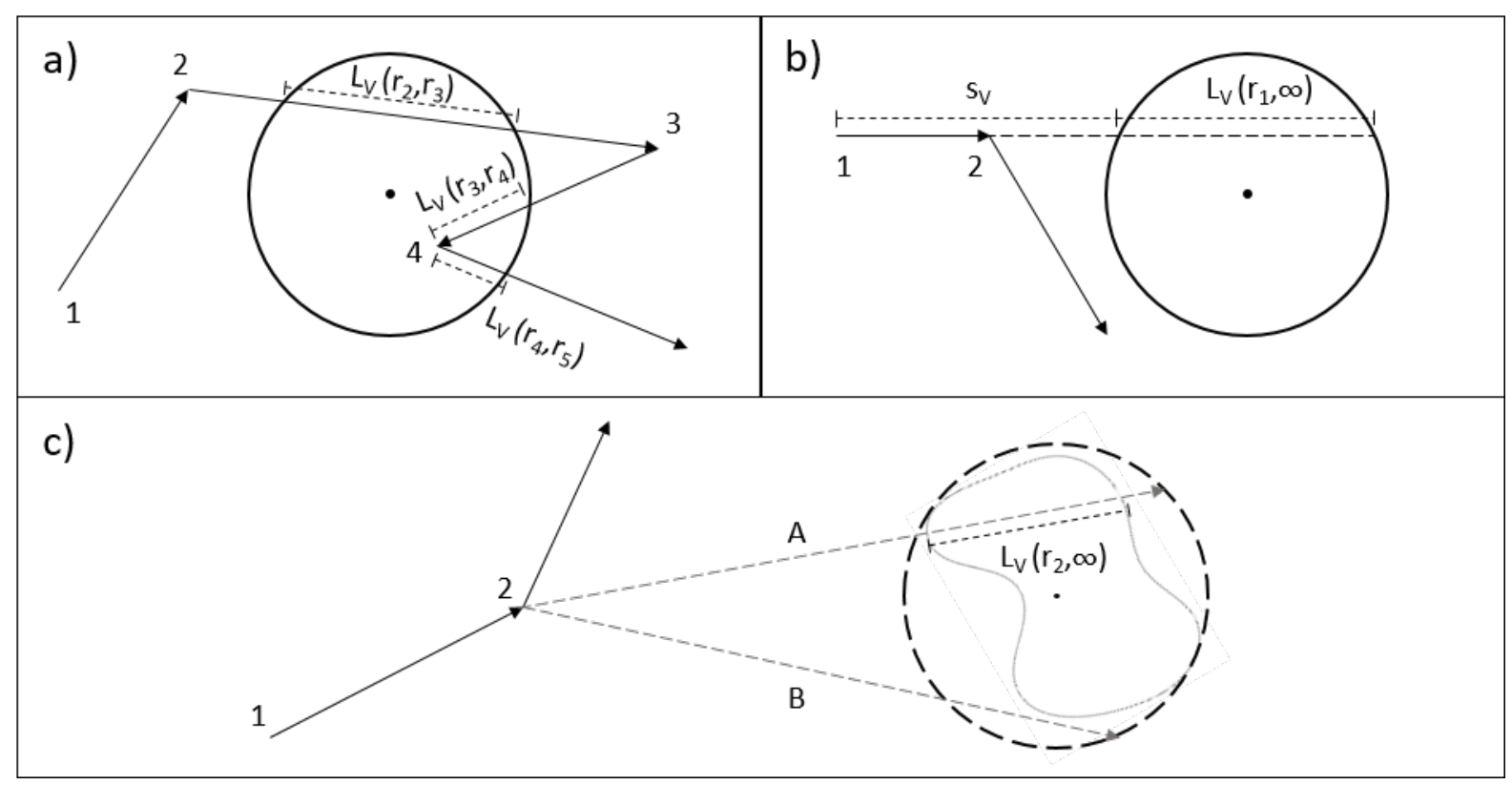

Fig. 1. Visual representation of different track-length estimators: a) linear TLE; b) exponential TLE or $e$ TLE; c) $e$ TLE with forced detection algorithm

\section{2 eTLE implementation in TRIPOLI-4 ${ }^{\circledR}$}

We will now describe how the $e$ TLE with FD algorithm is implemented in TRIPOLI- ${ }^{\circledR}$. The algorithm takes place immediately after the calculation of the random free flight of the particle, when the particle's interaction has not yet been sampled. Since the estimator acts on a "virtual" particle trajectory, the first step consists in creating a fictive particle, which retains the original particle's direction of motion, energy, and simulation weight. The fictive particle immediately undergoes a fictive collision which sends it towards the detector volume, and is then killed after the score. The detailed implementation (see also fig. 2) is as follows:

1. First, the user associates a bounding sphere to each detection volume. The sphere must fully enclose the detection volume while still being as small as possible for maximum efficiency.

2. If the simulation has more than one detector, we start a loop over all of them.

3. We create a "virtual" particle as an exact copy of the original particle.

4. Based on the bounding radius and the distance between the interaction point and the sphere center, we calculate the solid angle subtended by the sphere, $\Delta \Omega$. Then, we sample a random direction uniformly distributed within $\Delta \Omega$. 
5. The code samples a "virtual" collision for the fictive particle, adjusting its weight (for implicit capture and for the scattering probability, through the factor $p\left(\boldsymbol{r}_{j}, \boldsymbol{\Omega}\right) \Delta \Omega$ ) and assigning the direction determined at the previous step, effectively directing the particle towards the bounding sphere. The energy of the fictive particle is sampled by taking into account the differential cross-section of the collision, according to the direction randomly selected at point 4 . This ensures that the physics of the simulation remains unbiased.

6. We calculate the straight-line distance $d_{j}$ between the virtual particle and the closest volume frontier.

7. The virtual particle is displaced to its new position on the volume frontier; a value $k_{j}=w_{j-1}\left(1-e^{-\Sigma_{t} d_{j}}\right) / \Sigma_{t}$ is stored in memory for the volume just crossed. Here, $w_{j-1}$ is the weight of the virtual particle before the $j$-th displacement and $\Sigma_{t}$ is the total macroscopic cross section in the traversed volume. Therefore $w_{j_{1}}\left(1-e^{-\Sigma_{t} d_{j}}\right)$ represents the weight loss due to attenuation, and $1 / \Sigma_{t}$ is the expected value of the particle's free flight in the medium.

8. The weight of the particle $w_{j}$ is updated: $w_{j}=w_{j-1} e^{-\Sigma_{t} d_{j}}$.

9. We go back to point 6 and repeat the process until the virtual particle reaches a domain boundary (i.e., until it leaves the geometric domain of the simulation).

10. If the particle has crossed the detector volume, we register the score calculated at point 7 . Then, we kill the particle and go back to point 2 , selecting the next detector in the list.

11. Finally, the virtual particle is deleted and all transport quantities are reinitialized, before going back to the transport of the "real" particle.

At the end of the process, the quantity searched can be calculated for the detector volume starting from $k_{j}$. For example, if the quantity under consideration is the absorbed dose in the volume $D_{j, V}$, the result is given by:

$$
D_{j, V}=\frac{k_{j} E_{j} \frac{\mu_{e n}}{\rho}}{V}
$$

where $E_{j}$ is the photon's energy, $\mu_{e n} / \rho$ is the mass energy absorption coefficient of the material, and $V$ is the detector's volume.

\subsection{Simulation setup and test cases}

This section presents the various geometrical configurations that have been tested to estimate the performance of $e$ TLE, and the general setup of the MC simulations. For each configuration, the estimator was tested with and without the forced detection algorithm and compared to the well validated linear TLE of TRIPOLI-4 ${ }^{\circledR}$. In all simulations, ${ }^{60} \mathrm{Co}$ is used as a photon source, emitting photons at energies of $1.33 \mathrm{MeV}$ and $1.17 \mathrm{MeV}$ with an intensity of $1.59 \times 10^{10}$ photons/s. The calculated quantity is the KERMA rate, which is equal to the absorbed dose rate in conditions of charged particle equilibrium. Electron and positron transport is deactivated, therefore all energy related to these particles is assumed to be deposited locally. The configurations studied are fairly simple and only include the following four materials, all assumed to be at room temperature (weight percentages are indicated):

- Air: $77 \%$ N, $23 \%$ O, $0.00128 \mathrm{~g} / \mathrm{cm}^{3}$

- Concrete: $54 \%$ O, $34 \% \mathrm{Si}, 4 \% \mathrm{Ca}, 3.5 \% \mathrm{Al}, 1.5 \% \mathrm{Fe}, 1.5 \% \mathrm{H}, 1.5 \% \mathrm{Na}, 2.3 \mathrm{~g} / \mathrm{cm}^{3}$

- Lead: $100 \% \mathrm{~Pb}, 11.35 \mathrm{~g} / \mathrm{cm}^{3}$

- Iron: $100 \% \mathrm{Fe}, 7.85 \mathrm{~g} / \mathrm{cm}^{3}$

Batches of 10000 photons are used. The total number of batches for each simulation may vary, as the stopping criterion is defined as a limit on the maximum statistical uncertainty $\sigma$ over all detectors, which will be detailed below for every configuration. The machine used for all simulations is equipped with two Intel Xeon E5-2620v3 processors. A figure of merit $(F O M)$ is defined for each detector volume as:

$$
F O M=\frac{1}{t \sigma^{2}}
$$

where $t$ is the total simulation time and $\sigma^{2}$ is the variance of the dose rate estimated in the detector, expressed in percent. Additional geometrical and simulation details for each configuration are given in the following.

\subsubsection{Back-scattering configuration}

The first configuration reproduces a set of shielding benchmarks originally performed by Odano et al. [25]. The backscattering configuration (fig. 3a) assesses the performance of an estimator concerning the phenomenon of photon back-scattering, or reflection, on concrete. The direct path between the ${ }^{60} \mathrm{Co}$ point-wise source and the detector 


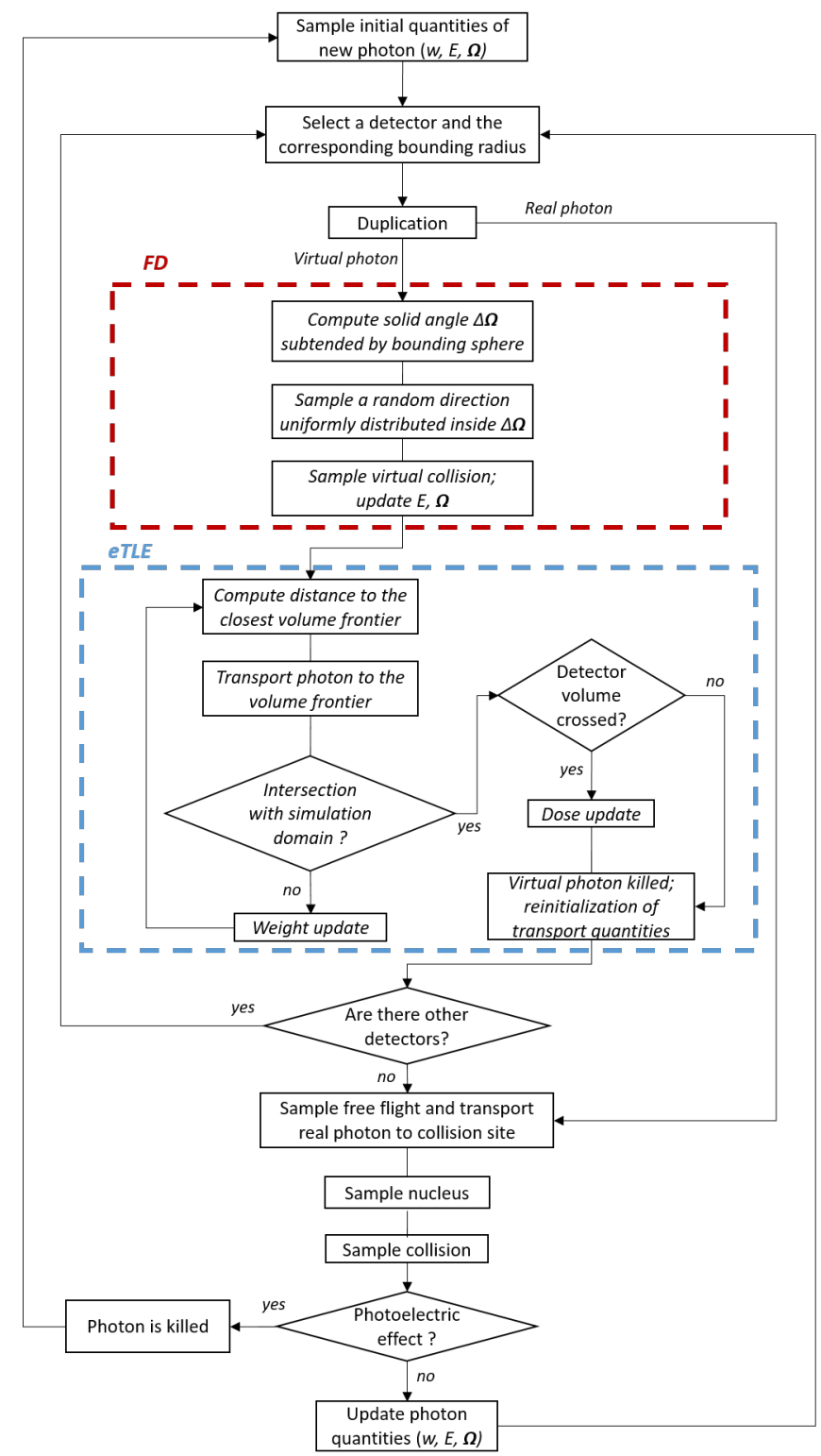

Fig. 2. Flowchart of the $e$ TLE algorithm with forced detection; $w$ is the particle's weight, $E$ is its energy and $\Omega$ is its direction.

volumes is shielded by a lead slab of $10 \times 40 \times 5 \mathrm{~cm}^{3}$. A concrete slab measuring $100 \times 100 \times 20 \mathrm{~cm}^{3}$ is placed at a distance of $50 \mathrm{~cm}$ from the photon source, allowing photons to scatter towards the detectors bypassing the shield. The five detectors are modeled as $1 \mathrm{~cm}$ radius spheres spaced $5 \mathrm{~cm}$ from each other. The maximum target $\sigma$ for the simulation is set at $5 \%$.

\subsubsection{Slant penetration configuration}

The slant penetration configuration (fig. 3b) determines the estimator response to photons traveling inside an attenuating medium with increasing slant angles. The attenuating medium is a $100 \times 200 \times 20 \mathrm{~cm}^{3}$ concrete slab placed between photon source and detectors at a distance of $1 \mathrm{~cm}$ from either side. The first detector is placed vertically above the source; the remaining ten are spaced $10 \mathrm{~cm}$ from each other, creating increasing slant angles with respect to the source, up to an angle of about $78^{\circ}$. The detectors are modeled as $1 \mathrm{~cm}$ radius spheres. The maximum target $\sigma$ for the simulation is set at $5 \%$. 


\subsubsection{Streaming duct configuration}

The streaming duct configuration (fig. 4a) tests the performance of the estimator in the case of a streaming problem, i.e. an empty tube traversing a scattering medium thus creating a preferential way for particle transport. The pointwise isotropic ${ }^{60} \mathrm{Co}$ source is placed in a lead collimator which effectively turns it into a conical-emitting source. A concrete structure with an empty steel tube traversing it is placed at a distance of two meters from the source with a $45^{\circ}$ angle. Eighteen $1 \mathrm{~cm}$ radius spherical detectors are placed along the duct at six different axial positions. Each axial position has three detectors, spaced $7.5 \mathrm{~cm}$ from each other. The maximum target $\sigma$ for the simulation is set at $5 \%$.

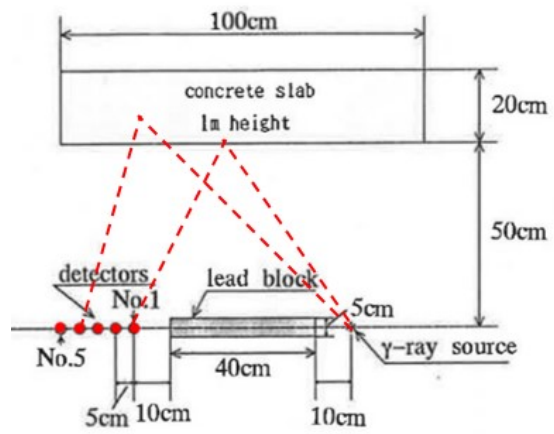

a) Back-scattering configuration

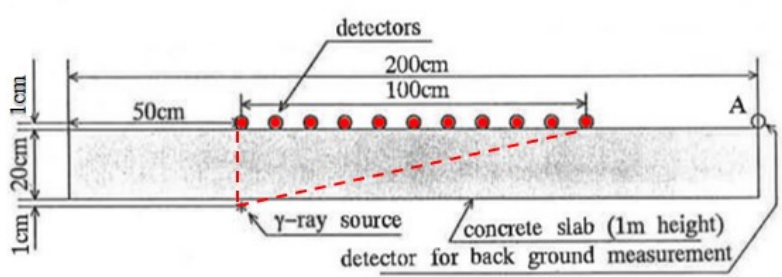

a) Slant penetration configuration

Fig. 3. Geometry of the back-scattering (a) and slant penetration (b) configurations - Red points represent the detector locations, red lines represent some of the possible photon paths.

\subsubsection{Bunker configuration}

The bunker configuration (fig. 4b) aims at condensing all the relevant ways of photon transport (scattering, penetration, streaming) in a unified and simple geometrical setup. It represents a typical shielding problem, with a photon source placed in an empty room with scattering concrete walls and a highly-absorbing lead shield partially covering the detector. The bunker's internal dimensions are $1 \times 1 \times 1 \mathrm{~m}^{3}$, with concrete walls measuring $20 \mathrm{~cm}$ in thickness; the detector volume is a $10 \mathrm{~cm}$ radius sphere and the lead shield, placed at $50 \mathrm{~cm}$ from the source, varies in height and in depth. A total of six configurations were studied, with different shield dimensions as described in table 1 . The maximum target $\sigma$ for the simulation is set at $1 \%$.

Table 1. Shield height (h) and depth (d) variation for bunker configuration

\begin{tabular}{ccc}
\hline \hline Name & $\mathrm{h}(\mathrm{cm})$ & $\mathrm{d}(\mathrm{cm})$ \\
\hline \hline R1 & 50 & 10 \\
R2 & 80 & 10 \\
R3 & 100 & 10 \\
R4 & 50 & 20 \\
R5 & 80 & 20 \\
R6 & 100 & 20 \\
\hline \hline
\end{tabular}

\section{Results}

\subsection{Validation}

This step serves to ensure that the new estimator converges to the same results as the classic TLE already implemented in TRIPOLI- ${ }^{\circledR}$. The dose rate computed by $e$ TLE with and without the FD algorithm is compared with the dose rate 


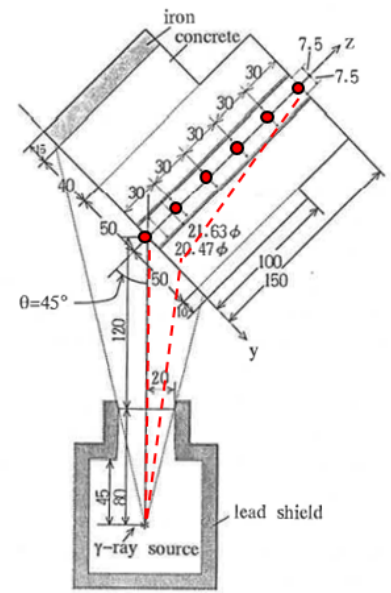

a) Streaming duct configuration

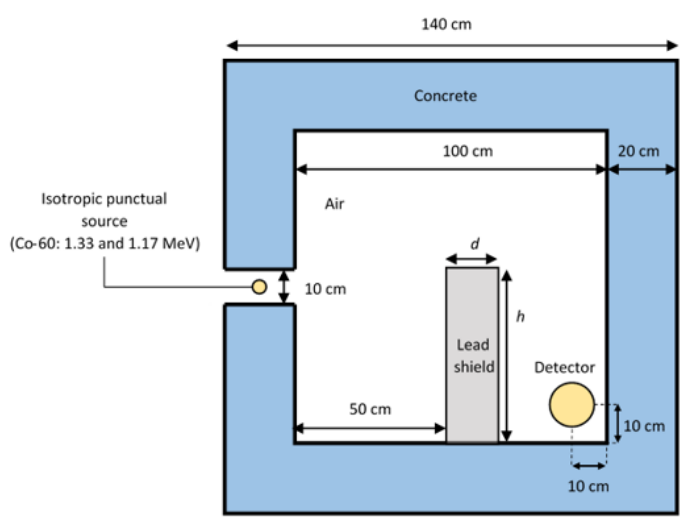

b) Bunker configuration

Fig. 4. Geometry of the streaming duct (a) and bunker (b) configurations - Red points represent the detector locations, red lines represent some of the possible photon paths.

obtained with the TLE. Fig. 5 shows the ratio between the two estimates, with tolerance margins of $\pm 2 \sigma$ highlighted on the plots. The tolerance was chosen according to the central limit theorem: an interval of $\pm 2 \sigma$ includes the exact simulation result with a probability of $95.45 \%$ Each point on the plots shows the dose rate of a single detector, with the exception of the streaming duct case, where results for each triplet of detectors occupying the same axial position were averaged to improve legibility. In general, we note a very good accordance with the TLE results: all results fall inside the error margin, if we consider their relative uncertainty. Both versions of the $e$ TLE, with and without FD, are therefore validated.

a) Slant penetration

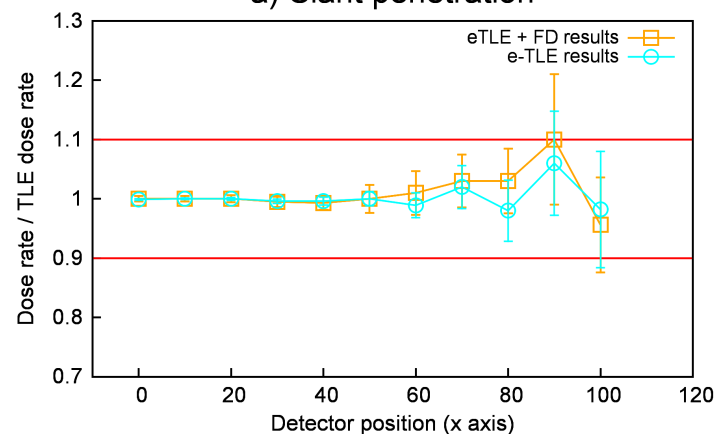

c) Backscattering

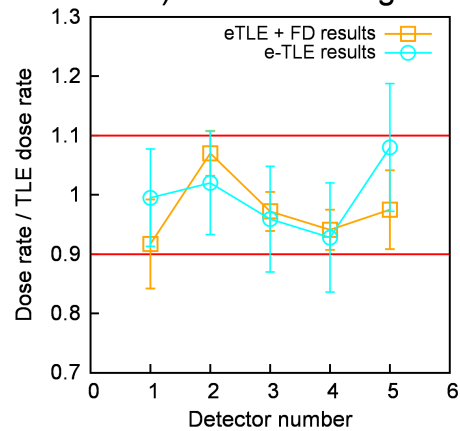

d) Bunker, $d=10 \mathrm{~cm}$

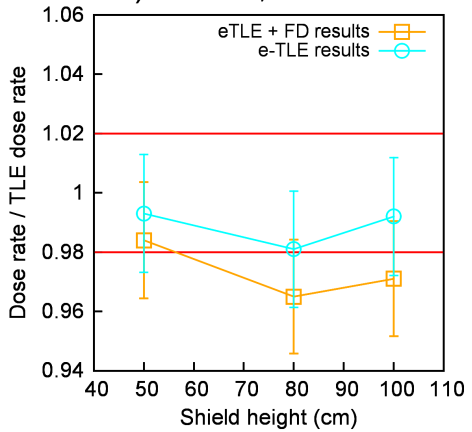

b) Streaming duct

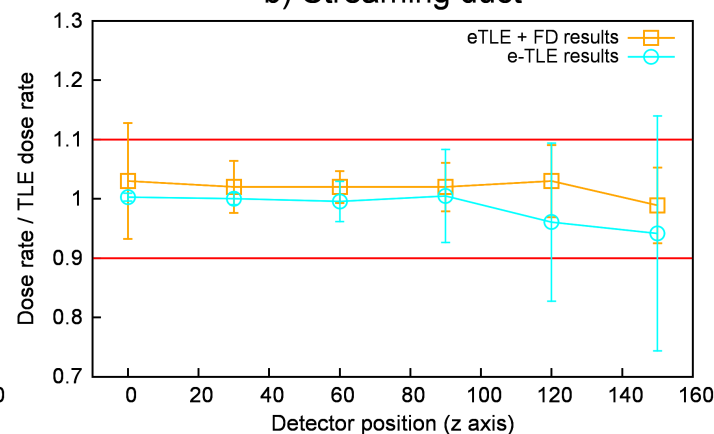

e) Bunker, $d=20 \mathrm{~cm}$

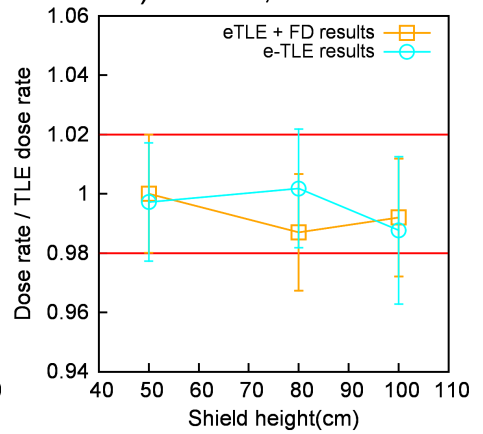

Fig. 5. Validation of the estimator over all tested configurations. The y axis shows the ratio between the equivalent dose rate calculated with the $e$ TLE estimator (with and without forced detection) and the one calculated with TRIPOLI- ${ }^{\circledR}$ 's TLE 


\subsection{Performance of $e$ TLE}

To assess the performance of the new estimator, we compare in fig. 6 the FOM of the simulations with the $e$ TLE to the FOM of the simulations performed with the normal track-length estimator. Therefore, a ratio higher than 1 means that the new exponential estimator performs better than the classic linear one.

In the back-scattering configuration (fig. 6c) the $e$ TLE with FD attains outstanding performances, with acceleration factors up to $2 \times 10^{4}$. This is an enormous improvement with respect to the normal $e$ TLE, which has acceleration ratios around 1.2 for this configuration.

In the bunker configuration (fig. $6 \mathrm{~d}$ and $6 \mathrm{e}$ ), all simulations are accelerated by the $e$ TLE with and without FD. Specifically, with the FD algorithm we reach factors of almost 100, while the normal $e$ TLE had a factor of 2 in the best case.

Results are more varied in the slant penetration (6a) and streaming configurations (6b). In the slant penetration setup, we see that the eTLE without FD is able to uniformly accelerate the results over all the detectors, with acceleration ratios of about 2 . With the FD algorithm, the estimator shows increasingly good performances as we move further from the source, with acceleration ratios ranging from lower than 1 (which indicates a drop in performance with respect to the TLE) to around 10 . We can find the same behavior in the streaming configuration, with the estimator failing to accelerate the convergence over the first two detectors and progressively increasing its performances as we move to the farthest detectors, with ratios close to 30 . This is also the only configuration where the $e$ TLE without FD fails to accelerate the convergence.

a) Slant penetration

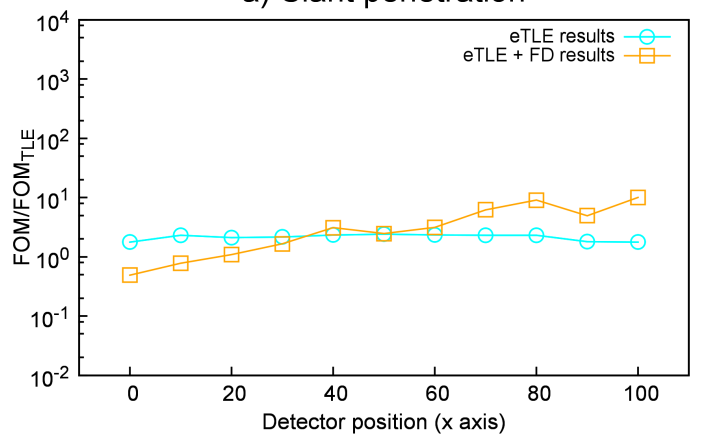

c) Back-scattering

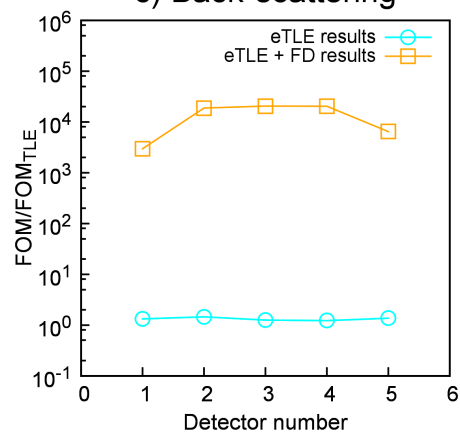

d) Bunker, $d=10 \mathrm{~cm}$

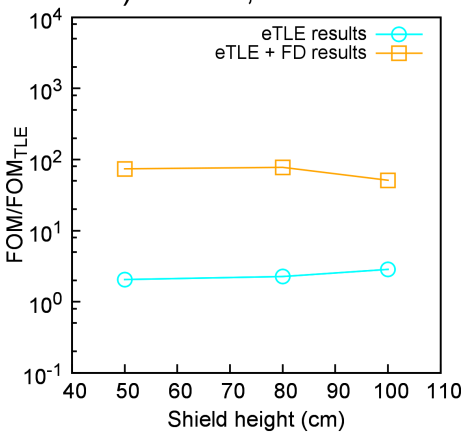

b) Streaming duct

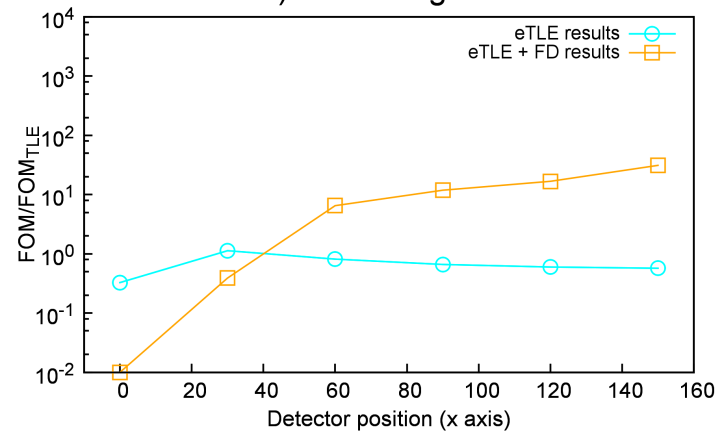

e) Bunker, $d=20 \mathrm{~cm}$

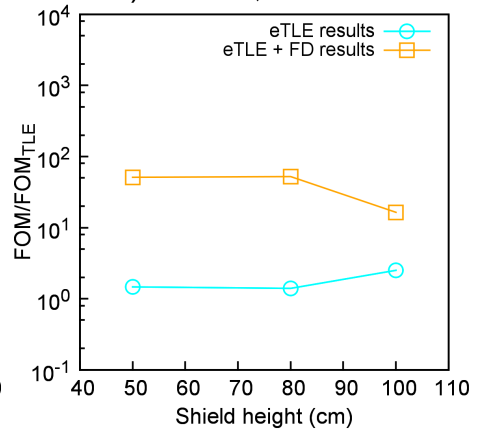

Fig. 6. Performance of the estimator over all tested configurations. The y axis shows the ratio between the figure of merit of the $e$ TLE estimator (with and without forced detection) and the figure of merit of TRIPOLI- ${ }^{\circledR}$ 's TLE

An explanation for this behavior might be found in the fact that having to simulate and transport virtual particles adds a certain amount of overhead to the transport algorithm. To generate a single particle history, the $e$ TLE with FD requires the largest amount of time (which increases with the total number of detectors), followed by the normal $e$ TLE and then by the classic TLE. This of course means that, given a certain amount of computation time, the simulation with the TLE will generate a larger amount of particle histories than the one using the $e$ TLE. Therefore, if a detector is close to the source and easily reached by the particles, convergence with the TLE will be quicker.

As we mentioned before, in the case of multiple detectors, the current implementation of the FD algorithm sends one virtual particle to each of the detectors before restarting the original particle transport. Intuitively, this implies that the algorithm's efficiency is inversely proportional to the number of detectors in the simulation. Therefore, to try and improve the estimator's performance, we ran an additional set of simulations on both the slant penetration and the streaming duct configurations. In this case, only one detector was active, which means that a total of 22 
simulations were run in the slant penetration case (11 with the TLE, 11 with the $e$ TLE) and 12 simulations were run in the streaming case ( 6 with the TLE, 6 with the $e$ TLE). Results of this "single detector" simulations are reported in fig. 7. As we can see from the plots, the estimator performance is drastically improved when used with only one detector, with the efficiency consistently gaining one order of magnitude with respect to the multiple-detector case. We can still see the same behavior related to the detector's distance from the source: for the farthest detectors, we register acceleration ratios of around 500 for the slant penetration case and more than 600 for the streaming duct.

a) Dose comparison, slant penetration

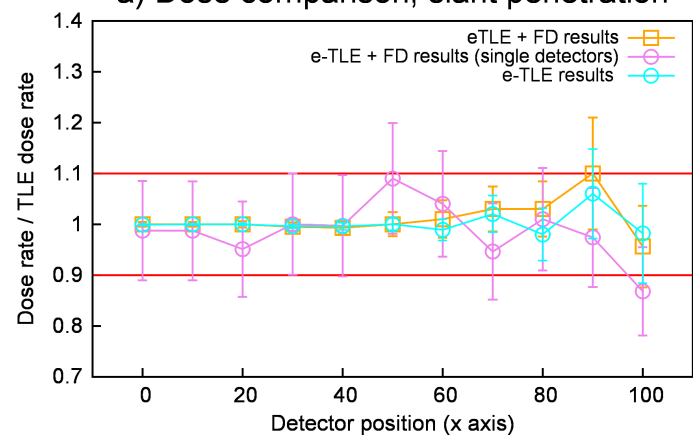

c) Dose comparison, streaming duct

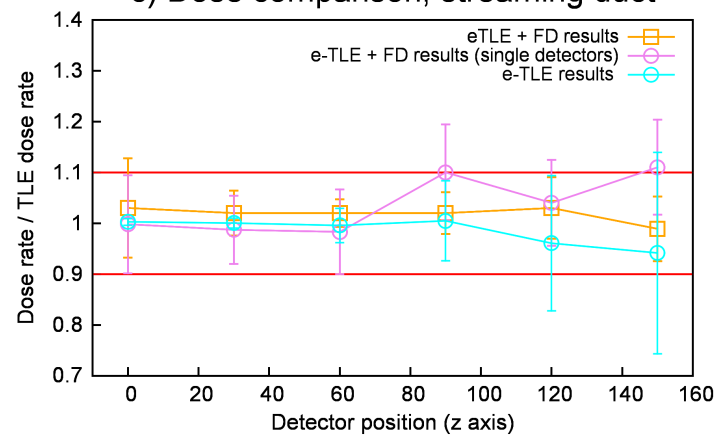

Fig. 7. Validation and performance of the estimator in single-detector simulations.

\section{b) Acceleration ratio, slant penetration}

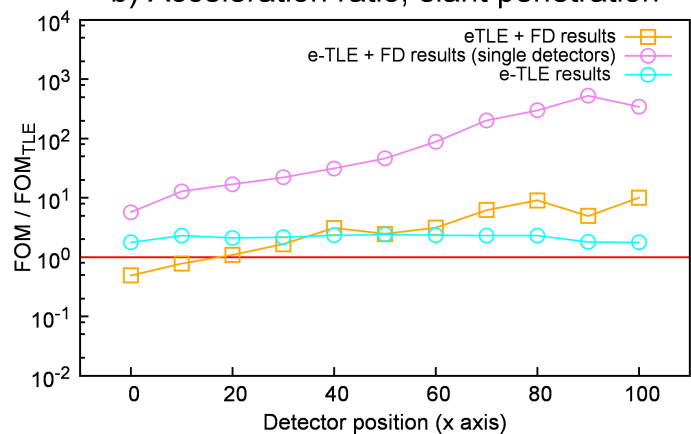

d) Acceleration ratio, streaming duct

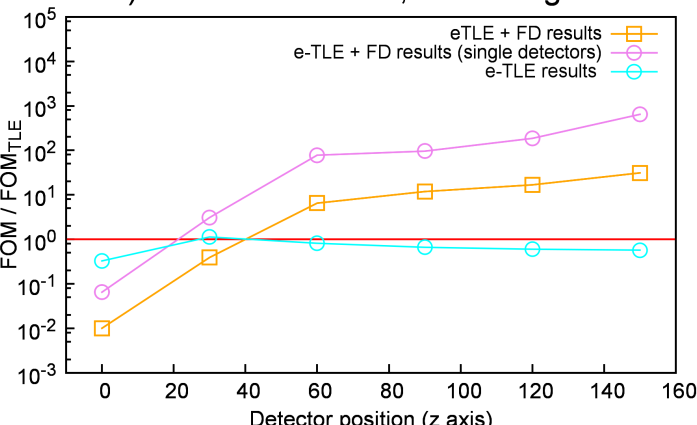

\section{Conclusions}

This paper provides a closer look at the $e$ TLE recently implemented in TRIPOLI-4 ${ }^{\circledR}$, with a particular focus on its use with a forced detection algorithm. The new estimator is validated, as it shows good statistical agreement with the results of the classic TLE of TRIPOLI-4 ${ }^{\circledR}$. Moreover, the estimator shows excellent acceleration capabilities when dealing with photon transport in void and scattering-intensive environments, with acceleration factors reaching up to $10^{4}$. The estimator shows the best performance when used with few detectors, especially if they are sufficiently far from the photon source. These first results are encouraging and open the way to future applications of the new estimator. Studies on the application of eTLE to neutron transport as well as photons are already being conducted, as well as a new mesh-scoring implementation which could significantly expand the estimator's domain of application.

\section{References}

1. P.C. Miller. RANKERN - a point kernel integration code for complicated geometry problems. Japan Atomic Energy Research Inst, Japan, 1983. INIS Reference Number: 16031167.

2. Thierry Visonneau, Laurence Pangault, Fadhel Malouch, Fausto Malvagi, and Florence Dolci. NARMER-1: a photon point-kernel code with build-up factors. EPJ Web of Conferences, 153:06028, 2017.

3. Christopher John Werner, Jeffrey S. Bull, C. J. Solomon, Forrest B. Brown, Gregg Walter McKinney, Michael Evan Rising, David A. Dixon, Roger Lee Martz, Henry G. Hughes, Lawrence James Cox, Anthony J. Zukaitis, J. C. Armstrong, Robert Arthur Forster, and Laura Casswell. MCNP Version 6.2 Release Notes. Technical Report LA-UR-18-20808, 1419730, February 2018. 
4. S. Agostinelli, J. Allison, K. Amako, J. Apostolakis, H. Araujo, P. Arce, M. Asai, D. Axen, S. Banerjee, G. Barrand, F. Behner, L. Bellagamba, J. Boudreau, L. Broglia, A. Brunengo, H. Burkhardt, S. Chauvie, J. Chuma, R. Chytracek, G. Cooperman, G. Cosmo, P. Degtyarenko, A. Dell'Acqua, G. Depaola, D. Dietrich, R. Enami, A. Feliciello, C. Ferguson, H. Fesefeldt, G. Folger, F. Foppiano, A. Forti, S. Garelli, S. Giani, R. Giannitrapani, D. Gibin, J. J. Gómez Cadenas, I. González, G. Gracia Abril, G. Greeniaus, W. Greiner, V. Grichine, A. Grossheim, S. Guatelli, P. Gumplinger, R. Hamatsu, K. Hashimoto, H. Hasui, A. Heikkinen, A. Howard, V. Ivanchenko, A. Johnson, F. W. Jones, J. Kallenbach, N. Kanaya, M. Kawabata, Y. Kawabata, M. Kawaguti, S. Kelner, P. Kent, A. Kimura, T. Kodama, R. Kokoulin, M. Kossov, H. Kurashige, E. Lamanna, T. Lampén, V. Lara, V. Lefebure, F. Lei, M. Liendl, W. Lockman, F. Longo, S. Magni, M. Maire, E. Medernach, K. Minamimoto, P. Mora de Freitas, Y. Morita, K. Murakami, M. Nagamatu, R. Nartallo, P. Nieminen, T. Nishimura, K. Ohtsubo, M. Okamura, S. O'Neale, Y. Oohata, K. Paech, J. Perl, A. Pfeiffer, M. G. Pia, F. Ranjard, A. Rybin, S. Sadilov, E. Di Salvo, G. Santin, T. Sasaki, N. Savvas, Y. Sawada, S. Scherer, S. Sei, V. Sirotenko, D. Smith, N. Starkov, H. Stoecker, J. Sulkimo, M. Takahata, S. Tanaka, E. Tcherniaev, E. Safai Tehrani, M. Tropeano, P. Truscott, H. Uno, L. Urban, P. Urban, M. Verderi, A. Walkden, W. Wander, H. Weber, J. P. Wellisch, T. Wenaus, D. C. Williams, D. Wright, T. Yamada, H. Yoshida, and D. Zschiesche. Geant4-a simulation toolkit. Nuclear Instruments and Methods in Physics Research Section A: Accelerators, Spectrometers, Detectors and Associated Equipment, 506(3):250-303, July 2003.

5. E. Brun, F. Damian, C. M. Diop, E. Dumonteil, F. X. Hugot, C. Jouanne, Y. K. Lee, F. Malvagi, A. Mazzolo, O. Petit, J. C. Trama, T. Visonneau, and A. Zoia. TRIPOLI-4@, CEA, EDF and AREVA reference Monte Carlo code. Annals of Nuclear Energy, 82:151-160, August 2015.

6. F Vermeersch and C Van Bosstraeten. Development of the VISIPLAN ALARA planning tool. In Proceeding of the International Conference on Topical issues in Nuclear Radiation and Radioactive Waste Safety, page 6, Vienna, Austria, September 1998.

7. Canberra Industries. MERCURAD - Dose Rate Calculation Software, User's Manual. Technical Report DEX-DT-61316, 2003.

8. I. Szóke, M. N. Louka, T. R. Bryntesen, J. Bratteli, S. T. Edvardsen, K. K. RøEitrheim, and K. Bodor. Real-time 3D radiation risk assessment supporting simulation of work in nuclear environments. Journal of Radiological Protection: Official Journal of the Society for Radiological Protection, 34(2):389-416, June 2014.

9. Matthieu Longeot, Bruno Dupont, Mark Zweers, Fausto Malvagi, Jean-Christophe Trama, and Julien Dubost. PANTHERE: simulation software for 3D dose rate calculation in complex nuclear facilities. Progress in Nuclear Science and Technology, 4:557-560, 2014.

10. G. Gualdrini and P. Ferrari. Monte Carlo variance reduction techniques: an overview with some practical examples. Radiation Protection Dosimetry, 146(4):425-433, July 2011.

11. Alireza Haghighat and John C. Wagner. Monte Carlo variance reduction with deterministic importance functions. Progress in Nuclear Energy, 42(1):25-53, January 2003.

12. Jeffrey F. Williamson. Monte Carlo evaluation of kerma at a point for photon transport problems: Monte Carlo calculation of kerma at a point. Medical Physics, 14(4):567-576, July 1987.

13. Robert J. Barish. The Dosimetry of Ionizing Radiation. Vol 3. Radiology, 180(1):120-120, July 1991.

14. F. Baldacci, A. Mittone, A. Bravin, P. Coan, F. Delaire, C. Ferrero, S. Gasilov, J.M. Létang, D. Sarrut, F. Smekens, and N. Freud. A track length estimator method for dose calculations in low-energy X-ray irradiations: implementation, properties and performance. Zeitschrift für Medizinische Physik, 25(1):36-47, March 2015.

15. Alberto Mittone, Fabien Baldacci, Alberto Bravin, Emmanuel Brun, François Delaire, Claudio Ferrero, Sergei Gasilov, Nicolas Freud, Jean Michel Létang, David Sarrut, François Smekens, and Paola Coan. An efficient numerical tool for dose deposition prediction applied to synchrotron medical imaging and radiation therapy. Journal of Synchrotron Radiation, 20(5):785-792, September 2013.

16. Tuomas Viitanen and Jaakko Leppänen. Validating the Serpent Model of FiR 1 Triga Mk-II Reactor by Means of Reactor Dosimetry. EPJ Web of Conferences, 106:03010, 2016. Publisher: EDP Sciences.

17. E. J. McGrath and D. C. Irving. Techniques for Efficient Monte Carlo Simulation. Volume 3. Variance Reduction:. Technical report, Defense Technical Information Center, Fort Belvoir, VA, March 1973.

18. N. Freud, J.-M. Létang, and D. Babot. A hybrid approach to simulate multiple photon scattering in X-ray imaging. Nuclear Instruments and Methods in Physics Research Section B: Beam Interactions with Materials and Atoms, 227(4):551-558, January 2005.

19. Nicolas Freud, Jean Michel Letang, Corentin Mary, Caroline Boudou, Claudio Ferrero, HÉlĖne Elleaume, Alberto Bravin, FranÇois Esteve, and Daniel Babot. A Hybrid Approach for Fast Simulation of Dose Deposition in Stereotactic Synchrotron Radiotherapy. IEEE Transactions on Nuclear Science, 55(3):1008-1017, June 2008.

20. David R. Haynor, Robert L. Harrison, and Thomas K. Lewellen. The use of importance sampling techniques to improve the efficiency of photon tracking in emission tomography simulations. Medical Physics, 18(5):990-1001, 1991. _eprint: https://aapm.onlinelibrary.wiley.com/doi/pdf/10.1118/1.596615.

21. Ettore Guadagni, Y. Pénéliau, Jean Michel Létang, François-Xavier Hugot, and Cindy Le Loirec. A hybrid deterministicstochastic method for the calculation of dose rate in reactor dismantling operations. In ANS MESC 2021 - The International Conference on Mathematics and Computational Methods Applied to Nuclear Science and Engineering, Raleigh, North Carolina, October 2021.

22. S. N. Cramer. Variance reduction methods applied to deep-penetration problems. Technical Report CONF-8410170-2, Oak Ridge National Lab., TN (USA), January 1984. 
23. J. S. Hendricks and T. E. Booth. MCNP variance reduction overview. In Raymond Alcouffe, Robert Dautray, Arthur Forster, Guy Ledanois, and B. Mercier, editors, Monte-Carlo Methods and Applications in Neutronics, Photonics and Statistical Physics, volume 240, pages 83-92. Springer-Verlag, Berlin/Heidelberg, 1985. Series Title: Lecture Notes in Physics.

24. J. R. Tickner. Algorithm for forcing scattered radiation to arbitrary convex regions in neutral particle Monte Carlo simulation. Nuclear Instruments and Methods in Physics Research Section B: Beam Interactions with Materials and Atoms, 267(14):2361-2364, July 2009.

25. Naoteru Odano, Toshimasa Miura, and Yuji Shindo. Experimental Assessment of Gamma-Ray Shielding Transport Calculations by Monte Carlo Calculation Code and Point-Kernel Code. In Proceedings of the 8th International Conference on Radiation Shielding, volume 1, pages 1301-1308, Arlington, Texas, April 1994. 